\title{
Liste préliminaire des abeilles de I'ancienne région Franche-Comté (Hymenoptera, Anthophila)
}

\author{
par Pierre TERRET* (Coord), Dominique MALÉCOT** (Coord), David GENOUD**, Éric DUFRÊNE**, \\ Matthieu AUBERT** \& Jean-Yves CRETIN* \\ Publication : juillet 2020
}

\begin{abstract}
Résumé. Ce travail représente la synthèse des connaissances actuelles sur les Hyménoptères Anthophiles franc-comtois. C'est un point de départ qui vise à établir la première liste globale des espèces d'abeilles sauvages, afin d'en évaluer la biodiversité. Initié fin 2016 , il repose sur une synthèse des données historiques contenues dans les collections des entomologistes locaux et sur celles du Muséum d'Histoire Naturelle de Besançon, élargie à celles obtenues depuis quelques décennies par des associations naturalistes (OPIE-FC, CBNFC-ORI, CEN-FC, Réserves Naturelles...), des institutions d'enseignement (LEGTA Lons-le-Saunier Mancy et LEGTA Edgar Faure Montmorot), un bureau d'étude (ECOJURA Environnement-Insectes) et par des prospections contemporaines ciblées. Les déterminations ont été vérifiées et validées en étroite collaboration avec les spécialistes de l'Observatoire des Abeilles, David GENOUD, Éric DUFRÊNE et Matthieu AUBERT ainsi que par Christophe PRAZ que nous remercions ici. Cette première évaluation rassemble donc 275 espèces pour la région : 189 doubistes, 55 haut-saônoises, 225 jurassiennes et 1 du Territoire de Belfort. Cette synthèse sera sans doute vite rendue obsolète par l'étude de collections encore non analysées et par des inventaires récents, en cours ou en projet. Elle vise à motiver les entomologistes de la région à collecter et à identifier des spécimens pour améliorer les connaissances et faciliter à terme la mise en place d'une stratégie de conservation des abeilles sauvages comtoises et, dans un avenir non déterminé, l'étendre à la nouvelle région Bourgogne - Franche-Comté.
\end{abstract}

\begin{abstract}
Preliminary list of the bees of the Franche-Comté region (Hymenoptera, Anthophila). This work synthesizes the knowledge of Hymenoptera Anthophila of our region, with the aim to establish the first list of species to assess regional biodiversity. Begun in 2016, it summarizes historical data contained in the collections of the Besançon Natural History Muséum, and extends to other data obtained throughout decades by multiple naturalist associations (OPIE-FC, CBNFC-ORI, CEN-FC, Réserves Naturelles...), educational institutions (LEGTA de Mancy et Montmorot, Lons-le Saunier, Jura), independent consultancy firms (ECOJURA Environnement-Insectes) and targeted contemporary research. Specific determinations were verified and validated in collaboration with several specialists, such as David GEnoud, Éric DUfRÊNE, Matthieu AUBERT and Christophe PraZ, to whom we would like to express here our gratitude. The present work shows the presence of 275 species from the former Franche-Comte region : 189 from the Doubs, 55 from the Haute-Saône, 225 from the Jura and 1 from the Territoire de Belfort. This synthesis will certainly become quickly obsolete, after analysis of collections not yet studied and more recent inventories. Nevertheless, it aims to encourage entomologists to prospect, collect and identify specimens to improve knowledge and enable the implementation of a conservation strategy for our wild bees. In the future, this works could be extended to the new region of Bourgogne - Franche-Comté.
\end{abstract}

Mots-clés. Hymenoptera, Anthophiles, inventaire, listes départementales. Key-words. Hymenoptera, Anthophila, bee inventory, species list.

\footnotetext{
* Office Pour les Insectes et leur Environnement de Franche-Comté (OPIE-FC) - 7, rue Voirin, 2500 Besançon pierre.terret@laposte.net - jean-yves.cretin@neuf.fr

** Observatoire des Abeilles (OA) - 68, rue du Onze Novembre, 59148 Flines-lez-Raches -

dominique.malecot@gmail.com - dge-davidgenoud@orange.fr - eric.dfn@wanadoo.fr - matthieu@oabeilles.net
} 


\section{Introduction}

Les Hyménoptères Apiformes jouent un rôle important dans la pollinisation des espèces végétales mais sont fortement menacés par l'intensification des pratiques agricoles (méthodes culturales, destruction de biotopes, raréfaction des ressources floricoles, pesticides...) qui contribue à l'uniformisation et à la disparition de leurs habitats. L'étude de HALLMANN et al. (2017) dans un réseau d'aires protégées allemandes met en évidence une diminution de la biomasse des insectes volants de $75 \%$ au cours des trente dernières années. Cette publication laisse penser que la perte de diversité serait générale et toucherait même les zones protégées, et que ce phénomène pourrait être très rapide dans ces populations d'insectes volants à effectifs artificiellement réduits. SANCHEZ-BAYO \& WYCKHUYS (2019) évoquent un déclin de $40 \%$ des espèces d'insectes, particulièrement les Hyménoptères.

À l'échelle franc-comtoise, l'autre modification d'habitats importante est celle des habitats forestiers. L'augmentation des surfaces forestières depuis le minimum forestier du XIXème siècle (LACHAUSSEE, 1954) et la mise en défens (réglementaire voire physique) de ces surfaces vis-à-vis de la dent des herbivores font que les ressources alimentaires, essentiellement associées à des floraisons en clairières et non directement sous couverts forestiers, se raréfient ou sont moins denses et équiréparties (faute de clairières). La disparition du pacage en forêt, la fermeture des milieux forestiers, des pré-bois et la simplification des lisières participent ainsi probablement à la perte de diversité pour ce groupe d'insectes.

Nous sommes peut-être proches d'atteindre un seuil de dégradation qui ne nous permettra plus de retrouver la diversité et les abondances que l'on connaissait auparavant, comme certains entomologistes de la région le pensent. Il est donc d'autant plus important de documenter les groupes d'invertébrés les moins connus et cela à un rythme plus soutenu que celui auquel ils disparaissent.

Nous proposons ici de la première liste validée d'abeilles sauvages connues dans l'ex-région Franche-Comté. Bien qu'il reste encore probablement plusieurs dizaines (voire centaines) d'espèces à (re)découvrir, nous proposons une liste déjà conséquente, avec 275 espèces recensées et validées, liste déclinée également par département. Notre souhait est de faire un premier état des lieux servant de base de connaissance, destinée à motiver les associations et les entomologistes locaux à l'étude de ce clade. L'historique du travail sur les Hyménoptères de la région et la méthode utilisée pour constituer ces listes d'espèces seront présentés. En conclusion, afin d'aider à cibler les futures prospections, les secteurs les moins connus et les perspectives de découvertes de nouvelles espèces seront ensuite discutés.

\section{Historique}

Avant le XXème siècle, la Franche-Comté n'était bien sûr pas un désert entomologique ! De nombreux amateurs avaient déjà investigué notre région, mais étaient surtout intéressés par les Coléoptères et les Lépidoptères, ayant amassé parfois des collections considérables en termes de diversité et d'intérêt majeur, ne serait-ce que par la présence d'endémiques remarquables... Des spécialistes des Hyménoptères pourtant fameux l'ont toutefois arpentée, mais n'ont pas laissé de traces du côté des Apiformes.

L'un des plus anciens est Jacques Ernest ANDRÉ (1838-1914), un Bourguignon né à Beaune devenu notaire et ayant passé toute son existence à Gray, en Haute-Saône, où il eut de nombreuses fonctions. Passionné par les Hyménoptères depuis son jeune âge, spécialiste des fourmis et des mutilles, il a rédigé avec son frère Edmond-Jacques Ernest ANDRÉ (1845-1891) le Species des Hyménoptères d'Europe \& d'Algérie, un monument de 11 tomes et plusieurs milliers de pages publié à la fin du XIX $^{\text {ème }}$ et au début du XX ${ }^{\text {ème }}$ siècle ! Il a chassé dans les environs de Gray et ses observations d'espèces sabulicoles* - mais recrutées chez les Sphécides, Pompilides et autres Vespides - ont été reprises par BERLAND $(1925,1928)$ et BERLAND \& BERNARD (1938), des espèces dont certaines ont été retrouvées à Quitteur dans une sablière qui est à l'heure actuelle gérée par le Conservatoire des Espaces Naturels de FrancheComté.

\footnotetext{
* Espèces sabulicoles : espèces qui effectuent tout ou partie de leur cycle de vie dans un substrat sableux.
} 
Il est impossible de penser que les entomologistes suisses n'ont jamais promené leurs filets dans notre région : Jacques DE BEAUMONT, amateur de Sphécidés (à qui l'on doit la Faune de Suisse), Auguste Forel, myrmécologue vaudois mondialement connu... À coup sûr, Francis BERNARD est passé chez nous, et bien d'autres. Mais rien qui puisse concerner les abeilles sauvages.

La première liste connue vient d'un inventaire entomologique global dans une pelouse sèche des environs de Besançon, réalisé en 1963 par JeanClaude ROBERT et publié en 1967, "Étude des hyménoptères aculéates d'une pelouse similixérothermique, éléments de biocénotique " (ROBERT, 1967). Ce travail avait été conduit sous la direction du Professeur Pierre REAL, enseignant en écologie du Laboratoire de biologie et écologie animales de la Faculté des Sciences de Besançon qui fédéra les entomologistes de la région au sein du Comité de liaison pour des recherches éco-faunistiques dans le Jura (CLERJ). Jean-Claude RoBERT et JeanYves CRETIN furent les premiers entomologistes, à notre connaissance, qui réalisèrent des inventaires d'abeilles (dans les années 1960 à 1995 environ). Jusqu'à la fin des années 90, en complément de chasses à vue dans différents sites de la région, l'utilisation de techniques telles que les " plateaux colorés " et les "pièges entomologiques composites " (PEC) (ROBERT, 1992 ; ROBERT, 1996) dans les aires protégées et les ZNIEFF a permis de collecter et d'identifier de nombreux spécimens d'abeilles.

Après un quasi-vide de près d'une décennie, trois inventaires utilisant des pièges fonctionnant toute la saison ont permis de faire un bond majeur dans la connaissance des Hyménoptères de la région et ceci grâce à l'appui technique de l'Observatoire des Abeilles (et notamment David GENOUD) concernant les identifications et les validations.

Ce sont chronologiquement :

- L'exploitation des collectes des tentes Malaise mises en place dans le Doubs pour l'inventaire syrphes des deux Réserves Naturelles Nationales du Ravin de Valbois et du lac de Remoray depuis 2009, dont au moins une a fait l'objet d'un rapport de synthèse (GENOUD \& LANGLOIS, 2013)

- Un inventaire par coupelles colorées conduit sur la périphérie de Lons-le-Saunier (39) dans le cadre du réseau Apiformes (collaboration Ministère de l'agriculture/INRA d'Avignon/ Observatoire des Abeilles) par le Lycée d'Enseignement Général et Technologique Agricole (LEGTA) Edgar Faure Montmorot et la Réserve Naturelle Régionale de la Côte de Mancy de 2010 à 2012 (LE FÉON et al., 2016)

- Enfin l'inventaire entomologique bénévole de Franche-Comté coordonné de 2011 à 2012 par le bureau d'étude ECOJURA EnvironnementInsectes avec la pose de pièges à vitres de grande dimension et de pièges Malaise dans trois sites de Franche-Comté (TERRET et al., 2012)

Une première liste de l'Arc jurassien (Ain, Jura, Doubs) non publiée a été réalisée par des membres de l'OA (GENOUd \& MALÉCOT, 2016) et a précédé ce travail à l'échelle franc-comtoise.

\section{Méthode}

Les abeilles forment un groupe dont la détermination est ardue au point que les entomologistes se spécialisent généralement sur un ou plusieurs genres. Le choix, pour cette liste, du clade des Anthophila dans la superfamille des Apoidea comme taxon cible repose sur l'existence d'entomologistes européens, français et locaux intéressés par du matériel et susceptibles d'identifier les spécimens ou de faire jouer le réseau de spécialistes avec qui ils travaillent. Les rencontres annuelles d'Apoidea Gallica sont la manifestation physique de ce réseau.

La liste repose notamment sur l'exploitation de la base de données régionales des invertébrés conjointe TAXA - OPIE-FC - CBNFC-ORI qui recueille les observations de l'entomofaune franccomtoise. Elle prend également appui sur les données de plusieurs autres structures que nous remercions : l'Observatoire des Abeilles (OA), l'INRA d'Avignon, le site Atlas Hymenoptera (Université de Mons, Belgique), deux Réserves Naturelles Nationales (Lac de Remoray, Ravin de Valbois) et une Réserve Naturelle Régionale (Côte de Mancy).

Lorsque la détermination n'était le fait que d'une personne, un retour au spécimen original était systématiquement demandé pour confrontation avec la détermination d'au moins une autre personne qualifiée. La recherche de données historiques contenues dans les collections privées et dans celles du Muséum d'Histoire Naturelle de 
Besançon a eu le même objectif : les spécimens en provenance de ces collections ont été systématiquement réévalués pour confirmation et validation des identifications originelles avant informatisation des données.

Chaque donnée (espèce/date/lieu) la plus récente et/ou la mieux validée selon les auteurs pour chaque département étudié a été associée au nom de son collecteur, à celui de son identificateur, à celui de son validateur et a été reliée, lorsque c'était pertinent, au projet d'inventaire dont elle était issue. Les échanges entre les participants (entomologistes locaux et spécialistes de l'OA) ont permis de valider les taxons présents à dire d'expert et d'arrêter la liste ci-après.

\section{Liste d'espèces / Résultats}

275 espèces sont dénombrées pour la région dont 189 pour le Doubs, 225 pour le Jura, 55 pour la Haute-Saône et 1 pour le Territoire de Belfort (Tableau I à VII, voir ci-dessous). À noter que la Haute-Saône (70) et le Territoire de Belfort (90) ont été largement sous-prospectés, ce qui explique le nombre d'espèces recensées moins important que dans les autres départements.

Tableau I. Nombre d'espèces d'Hyménoptères Anthophiles par famille et par département recensées en Franche-Comté

\begin{tabular}{|l|r|r|r|r|r|}
\hline \multicolumn{1}{|c|}{ Famille } & Doubs (25) & Jura (39) & $\begin{array}{c}\text { Haute-Saône } \\
\text { (70) }\end{array}$ & $\begin{array}{c}\text { Territoire de } \\
\text { Belfort (90) }\end{array}$ & $\begin{array}{c}\text { Total région } \\
\text { Franche- } \\
\text { Comté }\end{array}$ \\
\hline Andrénidés & 27 & 36 & 8 & 0 & 45 \\
\hline Apidés & 48 & 63 & 16 & 0 & 69 \\
\hline Colletidés & 13 & 13 & 3 & 0 & 18 \\
\hline Halictidés & 42 & 50 & 9 & 0 & 63 \\
\hline Mégachilidés & 54 & 58 & 16 & 1 & 73 \\
\hline Mélittidés & 5 & 5 & 3 & 0 & 7 \\
\hline Total espèces & 189 & 225 & 55 & 1 & $\mathbf{2 7 5}$ \\
\hline
\end{tabular}


Tableau II. Liste des espèces d'Hyménoptères Anthophiles par famille et par département recensées en Franche-Comté : ANDRENIDÉS

\begin{tabular}{|c|c|c|c|c|} 
Taxons & $\begin{array}{c}\text { Doubs } \\
\text { (25) }\end{array}$ & Jura (39) & $\begin{array}{c}\text { Haute- } \\
\text { Saône (70) }\end{array}$ & $\begin{array}{c}\text { Territoire } \\
\text { de Belfort } \\
\text { (70) }\end{array}$
\end{tabular}

Andrena alfkenella PERKINs, 1914

Andrena apicata SMITH, 1847

Andrena bicolor FABRICIUS, 1775

Andrena bucephala STEPHENS, 1846

Andrena carantonica PÉREZ, 1902

Andrena chrysosceles (KIRBY, 1802)

Andrena cineraria (LinNAEUS, 1758)

Andrena clarkella (KIRBY, 1802)

Andrena combinata (CHRIST, 1791)

Andrena congruens SCHMIEDEKNECHT, 1882

Andrena curvungula ThOMson, 1870

Andrena dorsata (KIRBY, 1802)

Andrena ferox SMITH, 1847

Andrena flavipes PANZER, 1799

Andrena fucata SMiTh, 1847

Andrena fulva (MÜLLER, 1766)

Andrena fulvago (CHRIST, 1791)

Andrena fulvata STOECKHERT, 1930

Andrena gravida IMHOFF, 1832

Andrena haemorrhoa (FABRICIUS, 1781)

Andrena hattorfiana (FABRICIUS, 1775)

Andrena helvola (L., 1758)

Andrena humilis ImHoFF, 1832

Andrena labiata FABRICIUS, 1781

Andrena lagopus LATREILLE, 1809

Andrena limata SMITH, 1853

Andrena minutula (KIRBY, 1802)

Andrena minutuloides PERKINs, 1914

Andrena nigroaenea (KIRBY, 1802)

Andrena nigroolivacea DouRs, 1873

Andrena nitida (MÜLleR, 1776)

Andrena pandellei PÉREZ, 1895

\begin{tabular}{|c|c|c|}
\hline $\mathrm{X}$ & & \\
\hline $\mathrm{X}$ & & \\
\hline $\mathrm{X}$ & $\mathrm{x}$ & \\
\hline & $\mathrm{X}$ & \\
\hline $\mathrm{X}$ & & \\
\hline $\mathrm{x}$ & $\mathrm{x}$ & $\mathrm{X}$ \\
\hline$x$ & $\mathrm{X}$ & \\
\hline $\mathrm{x}$ & $\mathrm{x}$ & \\
\hline $\mathrm{X}$ & & \\
\hline & $\mathrm{X}$ & \\
\hline $\mathrm{X}$ & $\mathrm{X}$ & \\
\hline $\mathrm{x}$ & $\mathrm{x}$ & \\
\hline & $\mathrm{X}$ & \\
\hline $\mathrm{x}$ & $\mathrm{X}$ & \\
\hline$x$ & $\mathrm{X}$ & \\
\hline $\mathrm{x}$ & $\mathrm{x}$ & $\mathrm{x}$ \\
\hline & $\mathrm{x}$ & \\
\hline $\mathrm{X}$ & $\mathrm{X}$ & \\
\hline & $\mathrm{X}$ & $\mathrm{X}$ \\
\hline $\mathrm{X}$ & $\mathrm{x}$ & \\
\hline $\mathrm{x}$ & & $\mathrm{X}$ \\
\hline & $\mathrm{X}$ & \\
\hline $\mathrm{x}$ & $\mathrm{x}$ & \\
\hline $\mathrm{x}$ & $\mathrm{x}$ & $\mathrm{X}$ \\
\hline & $\mathrm{x}$ & \\
\hline & $x$ & \\
\hline $\mathrm{x}$ & $\mathrm{x}$ & $\mathrm{X}$ \\
\hline $\mathrm{X}$ & $\mathrm{x}$ & \\
\hline $\mathrm{x}$ & & \\
\hline & $\mathrm{X}$ & \\
\hline $\mathrm{x}$ & $\mathrm{x}$ & \\
\hline $\mathrm{x}$ & & \\
\hline
\end{tabular}


Tableau II. Liste des espèces d'Hyménoptères Anthophiles par famille et par département recensées en Franche-Comté : ANDRENIDÉS

\begin{tabular}{|c|c|c|c|c|}
\hline Taxons & $\begin{array}{c}\text { Doubs } \\
\text { (25) }\end{array}$ & Jura (39) & $\begin{array}{c}\text { Haute- } \\
\text { Saône (70) }\end{array}$ & $\begin{array}{c}\text { Territoire } \\
\text { de Belfort } \\
\text { (70) }\end{array}$ \\
\hline
\end{tabular}

Andrena pilipes FABRICIUS, 1781

Andrena potentillae PANZER, 1809

Andrena proxima (KIRBY, 1802)

Andrena ranunculi SCHMIEDEKNECHT, 1883

Andrena rosae PANZER, 1801

Andrena rufula SCHMIEDEKNECHT, 1883

Andrena subopaca NYLANDER, 1848

Andrena vaga PANZER, 1799

Andrena varians (KIRBY, 1802)

Andrena viridescens VIERECK, 1916

Andrena wilkella (KIRBY, 1802)

Panurgus banksianus (KIRBY, 1802)

Panurgus calcaratus (ScOPOLI, 1763)

$$
\text { Région }=45 \text { espèces }
$$

\begin{tabular}{|c|c|c|c|}
\hline & $\mathrm{x}$ & & \\
\hline & $\mathrm{x}$ & & \\
\hline & $\mathrm{x}$ & & \\
\hline & $\mathrm{x}$ & & \\
\hline & $\mathrm{x}$ & & \\
\hline & $\mathrm{x}$ & & \\
\hline $\mathrm{x}$ & $\mathrm{x}$ & & \\
\hline $\mathrm{x}$ & $\mathrm{x}$ & & \\
\hline \multirow[t]{2}{*}{$\mathrm{x}$} & & & \\
\hline & $\mathrm{x}$ & & \\
\hline \multirow[t]{3}{*}{$\mathrm{x}$} & $\mathrm{x}$ & & \\
\hline & $\mathrm{x}$ & $\mathrm{x}$ & \\
\hline & & $\mathrm{x}$ & \\
\hline 27 & 36 & 8 & 0 \\
\hline
\end{tabular}

Tableau III. Liste des espèces d'Hyménoptères Anthophiles par famille et par département recensées en Franche-Comté : APIDÉS

\begin{tabular}{|c|c|c|c|c|} 
Taxons & $\begin{array}{c}\text { Houbs } \\
(25)\end{array}$ & Jura (39) & $\begin{array}{c}\text { Saône } \\
\text { (70) }\end{array}$ & $\begin{array}{c}\text { Territoire } \\
\text { de Belfort } \\
(70)\end{array}$
\end{tabular}

\begin{tabular}{|l|c|c|c|}
\hline Anthophora aestivalis (PANZER, 1801) & & $\mathrm{x}$ & $\mathrm{x}$ \\
\hline Anthophora furcata (PANZER, 1798) & $\mathrm{x}$ & $\mathrm{x}$ & \\
\hline Anthophora plumipes (PALLAS, 1772) & $\mathrm{x}$ & $\mathrm{x}$ & $\mathrm{x}$ \\
\hline Bombus barbutellus (KIRBY, 1802) & $\mathrm{x}$ & $\mathrm{x}$ & \\
\hline Bombus bohemicus SEIDL, 1838 & $\mathrm{x}$ & $\mathrm{x}$ & \\
\hline Bombus campestris (PANZER, 1801) & $\mathrm{x}$ & $\mathrm{x}$ & \\
\hline Bombus confusus SCHENCK, 1861 & $\mathrm{x}$ & $\mathrm{x}$ & \\
\hline Bombus cryptarum (FABRICIUS, 1775) & $\mathrm{x}$ & $\mathrm{x}$ & \\
\hline Bombus distinguendus MORAWITZ, 1869 & $\mathrm{x}$ & $\mathrm{x}$ & \\
\hline Bombus flavidus EVERSMANN, 1852 & & $\mathrm{x}$ & \\
\hline Bombus hortorum (L., 1761) & $\mathrm{x}$ & $\mathrm{x}$ & \\
\hline Bombus humilis ILLIGER, 1806 & $\mathrm{x}$ & $\mathrm{x}$ & \\
\hline
\end{tabular}


Tableau III. Liste des espèces d'Hyménoptères Anthophiles par famille et par département recensées en Franche-Comté : APIDÉS

\begin{tabular}{|c|c|c|c|c|}
\hline Taxons & $\begin{array}{c}\text { Doubs } \\
\text { (25) }\end{array}$ & Jura (39) & $\begin{array}{c}\text { Saône } \\
\mathbf{( 7 0 )}\end{array}$ & $\begin{array}{c}\text { Territoire } \\
\text { de Belfort } \\
\mathbf{( 7 0 )}\end{array}$ \\
\hline
\end{tabular}

Bombus hypnorum (L., 1758)

Bombus jonellus (KIRBY, 1802)

Bombus lapidarius (L., 1758)

Bombus lucorum (L., 1761)

Bombus mesomelas GERSTÄCKER, 1869

Bombus monticola SMITH, 1849

Bombus mucidus GERSTÄCKER, 1869

Bombus muscorum (L., 1758)

Bombus norvegicus (SPARRE-SCHNEIDER, 1918)

Bombus pascuorum (SCOPOLI, 1763)

Bombus pomorum (PANZER, 1805)

Bombus pratorum (L., 1761)

Bombus pyrenaeus PÉREZ, 1879

Bombus quadricolor (LEPELETIER, 1832)

Bombus ruderarius (MÜLLER, 1776)

Bombus ruderatus (FABRICIUS, 1775)

Bombus rupestris (FABRICIUS, 1793)

Bombus sichelii RADOSZKOWsKI, 1859

Bombus soroeensis (FABRICIUS, 1776)

Bombus subterraneus (L., 1758)

Bombus sylvarum (L., 1761)

Bombus sylvestris (LEPELETIER, 1832)

Bombus terrestris (L., 1758)

Bombus vestalis (GEOFFROY, 1785)

Bombus veteranus (FABRICIUS, 1793)

Bombus wurflenii RADOsZKowsKI, 1859

Ceratina cucurbitina (Rossi, 1792)

Ceratina cyanea (KIRBY, 1802)

Epeolus cruciger (PANZER, 1799)

Epeolus fallax MorawiTz, 1872

Eucera longicornis (L., 1758)

Eucera nigrescens PÉREZ, 1879

\begin{tabular}{|c|c|c|}
\hline $\mathrm{x}$ & $\mathrm{x}$ & \\
\hline & $\mathrm{x}$ & \\
\hline $\mathrm{x}$ & $\mathrm{x}$ & $\mathrm{x}$ \\
\hline $\mathrm{x}$ & $\mathrm{x}$ & \\
\hline $\mathrm{x}$ & $\mathrm{x}$ & \\
\hline & $\mathrm{x}$ & \\
\hline & $\mathrm{x}$ & \\
\hline $\mathrm{x}$ & & \\
\hline $\mathrm{x}$ & $x$ & \\
\hline $\mathrm{x}$ & $\mathrm{x}$ & $\mathrm{x}$ \\
\hline & $\mathrm{x}$ & \\
\hline $\mathrm{x}$ & $x$ & $\mathrm{x}$ \\
\hline & $\mathrm{x}$ & \\
\hline $\mathrm{x}$ & $\mathrm{x}$ & \\
\hline $\mathrm{x}$ & $\mathrm{x}$ & \\
\hline $\mathrm{x}$ & $\mathrm{x}$ & \\
\hline $\mathrm{x}$ & $x$ & \\
\hline & $\mathrm{x}$ & \\
\hline $\mathrm{x}$ & $\mathrm{x}$ & \\
\hline & $\mathrm{x}$ & \\
\hline$x$ & $\mathrm{x}$ & $\mathrm{x}$ \\
\hline $\mathrm{x}$ & $\mathrm{x}$ & \\
\hline$x$ & $\mathrm{x}$ & \\
\hline$x$ & $\mathrm{x}$ & \\
\hline $\mathrm{x}$ & $\mathrm{x}$ & \\
\hline$x$ & $\mathrm{x}$ & \\
\hline $\mathrm{x}$ & $\mathrm{x}$ & $\mathrm{x}$ \\
\hline $\mathrm{x}$ & $\mathrm{x}$ & $\mathrm{x}$ \\
\hline & $x$ & \\
\hline $\mathrm{x}$ & $\mathrm{x}$ & \\
\hline $\mathrm{x}$ & $x$ & \\
\hline $\mathrm{x}$ & $\mathrm{x}$ & $\mathrm{x}$ \\
\hline
\end{tabular}


Tableau III. Liste des espèces d'Hyménoptères Anthophiles par famille et par département recensées en Franche-Comté : APIDÉS

\begin{tabular}{|c|c|c|c|c|} 
Taxons & $\begin{array}{c}\text { Doubs } \\
(25)\end{array}$ & Jura (39) & $\begin{array}{c}\text { Saône } \\
\text { (70) }\end{array}$ & $\begin{array}{c}\text { Territoire } \\
\text { de Belfort } \\
\text { (70) }\end{array}$
\end{tabular}

\begin{tabular}{|c|c|c|c|c|}
\hline Melecta albifrons (FORSTER, 1771) & $\mathrm{x}$ & $\mathrm{x}$ & $\mathrm{x}$ & \\
\hline Nomada bifasciata OLIVIER, 1811 & $\mathrm{x}$ & $\mathrm{x}$ & & \\
\hline Nomada emarginata MoRAWITZ, 1877 & & $\mathrm{x}$ & & \\
\hline Nomada fabriciana (LINNAEUs, 1767) & $\mathrm{x}$ & $\mathrm{x}$ & $\mathrm{x}$ & \\
\hline Nomada femoralis MORAWITZ 1869 & & & $\mathrm{x}$ & \\
\hline Nomada flava PANZER, 1798 & $\mathrm{x}$ & $\mathrm{x}$ & & \\
\hline Nomada flavoguttata (KIRBY, 1802) & $\mathrm{x}$ & $\mathrm{x}$ & & \\
\hline Nomada fucata PANZER, 1798 & $\mathrm{x}$ & & & \\
\hline Nomada furva PANZER, 1798 & & $\mathrm{x}$ & & \\
\hline Nomada goodeniana (KIRBY, 1802) & $\mathrm{x}$ & $\mathrm{x}$ & & \\
\hline Nomada guttulata SCHENCK, 1861 & & $\mathrm{x}$ & & \\
\hline Nomada integra BRULLÉ, 1832 & & $\mathrm{x}$ & & \\
\hline Nomada lathburiana (KIRBY, 1802) & & $\mathrm{x}$ & & \\
\hline Nomada leucophthalma (KIRBY, 1802) & & $\mathrm{x}$ & & \\
\hline Nomada panzeri LEPELETIER, 1841 & $\mathrm{x}$ & & & \\
\hline Nomada ruficornis (L., 1758) & $\mathrm{x}$ & $\mathrm{x}$ & $\mathrm{x}$ & \\
\hline Nomada sexfasciata PANZER, 1799 & $\mathrm{x}$ & $\mathrm{x}$ & & \\
\hline Nomada signata JURINE, 1807 & $\mathrm{x}$ & & & \\
\hline Nomada striata FABRICIUS, 1793 & & $\mathrm{x}$ & & \\
\hline Nomada succincta PANZER, 1798 & & $\mathrm{x}$ & & \\
\hline Nomada tridentirostris DouRs, 1873 & & $\mathrm{x}$ & & \\
\hline Tetralonia malvae (RossI, 1790) & & $\mathrm{x}$ & $\mathrm{x}$ & \\
\hline Xylocopa iris (CHRIST, 1791) & $\mathrm{x}$ & & $\mathrm{x}$ & \\
\hline Xylocopa valga GERSTAECKER, 1872 & $\mathrm{x}$ & $\mathrm{x}$ & & \\
\hline Xylocopa violacea (L., 1758) & $\mathrm{x}$ & $\mathrm{x}$ & $\mathrm{x}$ & \\
\hline Région $=69$ espèces & 48 & 63 & 16 & 0 \\
\hline
\end{tabular}


Tableau IV. Liste des espèces d'Hyménoptères Anthophiles par famille et par département recensées en Franche-Comté : COLLETIDÉS

\begin{tabular}{|c|c|c|c|c|} 
Taxons & $\begin{array}{c}\text { Doubs } \\
(25)\end{array}$ & Jura (39) & $\begin{array}{c}\text { Haute- } \\
\text { Saône (70) }\end{array}$ & $\begin{array}{c}\text { Territoire } \\
\text { de Belfort } \\
\text { (70) }\end{array}$
\end{tabular}

\begin{tabular}{|c|c|c|c|c|}
\hline Colletes cunicularius (L., 1761) & $\mathrm{x}$ & $\mathrm{x}$ & $\mathrm{x}$ & \\
\hline Colletes daviesanus Sмітн, 1846 & & $\mathrm{x}$ & $\mathrm{x}$ & \\
\hline Colletes hederae SCHMIDT \& WESTRICH, 1993 & $\mathrm{x}$ & $\mathrm{x}$ & $\mathrm{x}$ & \\
\hline Colletes similis SCHENCK, 1853 & $\mathrm{x}$ & $\mathrm{x}$ & & \\
\hline Hylaeus angustatus (SCHENCK, 1861) & & $\mathrm{x}$ & & \\
\hline Hylaeus annularis (KIRBY, 1802) & $\mathrm{x}$ & & & \\
\hline Hylaeus brevicornis NYLANDER, 1852 & $\mathrm{x}$ & $\mathrm{x}$ & & \\
\hline Hylaeus communis NYLANDER, 1852 & $\mathrm{x}$ & $\mathrm{x}$ & & \\
\hline Hylaeus confusus NYLANDER, 1852 & $\mathrm{x}$ & $\mathrm{x}$ & & \\
\hline Hylaeus difformis (EVERSMANN, 1852) & $\mathrm{x}$ & & & \\
\hline Hylaeus dilatatus (KIRBY, 1802) & & $\mathrm{x}$ & & \\
\hline Hylaeus gibbus SAUNDERS, 1850 & $\mathrm{x}$ & & & \\
\hline Hylaeus gredleri FÖRSTER, 1871 & & $\mathrm{x}$ & & \\
\hline Hylaeus hyalinatus SMITH, 1842 & $\mathrm{x}$ & $\mathrm{x}$ & & \\
\hline Hylaeus kahri FöRSTER, 1871 & $\mathrm{x}$ & & & \\
\hline Hylaeus pictipes NYLANDER, 1852 & & $\mathrm{x}$ & & \\
\hline Hylaeus punctatus (BRULLÉ, 1832) & $\mathrm{x}$ & & & \\
\hline Hylaeus signatus (PANZER, 1798) & $\mathrm{x}$ & $\mathrm{x}$ & & \\
\hline Région $=18$ espèces & 13 & 13 & 3 & 0 \\
\hline
\end{tabular}


Tableau V. Liste des espèces d'Hyménoptères Anthophiles par famille et par département recensées en Franche-Comté : HALICTIDÉS

\begin{tabular}{c|c|c|c|c} 
& Doubs & Jura & $\begin{array}{c}\text { Haute- } \\
\text { Taxons }\end{array}$ & $\begin{array}{c}\text { Territoire } \\
\text { de Belfort }\end{array}$ \\
& $(25)$ & $(39)$ & $(70)$ & $(70)$
\end{tabular}

\begin{tabular}{|c|c|c|c|}
\hline Dufourea dentiventris (NYLANDER, 1848) & $\mathbf{x}$ & & \\
\hline Halictus aff. carinthiacus BLÜTHGEN, 1936 & & $\mathbf{x}$ & \\
\hline Halictus compressus (WALCKENAER 1802) & & $\mathbf{x}$ & \\
\hline Halictus langobardicus BLÜTHGEN, 1944 & & $\mathbf{x}$ & \\
\hline Halictus maculatus SMITH, 1848 & & $\mathbf{x}$ & \\
\hline Halictus quadricinctus (FABRICIUS, 1776) & $\mathbf{x}$ & & \\
\hline Halictus rubicundus (CHRIST, 1791) & $\mathbf{x}$ & $\mathbf{x}$ & \\
\hline Halictus scabiosae (Rossi, 1790) & $\mathbf{x}$ & $\mathbf{x}$ & $\mathbf{x}$ \\
\hline Halictus sexcinctus (FABRICIUS, 1775) & & $\mathbf{x}$ & $\mathbf{x}$ \\
\hline Halictus simplex BLÜTHGEN, 1923 & $\mathbf{x}$ & $\mathbf{x}$ & \\
\hline Halictus gr. tetrazonius (KLUG, 1817) & & $\mathbf{x}$ & \\
\hline Lasioglossum albipes (FABRICIUS, 1781) & $\mathbf{x}$ & $\mathbf{x}$ & \\
\hline Lasioglossum bluethgeni EBMER, 1971 & $\mathbf{x}$ & $\mathbf{x}$ & \\
\hline Lasioglossum calceatum (ScOPOLI, 1763) & $\mathbf{x}$ & $\mathbf{x}$ & \\
\hline Lasioglossum clypeare (SCHENCK, 1853) & $\mathbf{x}$ & & $\mathbf{x}$ \\
\hline Lasioglossum cupromicans (PÉREZ, 1903) & $\mathbf{x}$ & & \\
\hline Lasioglossum fulvicorne (KIRBY, 1802) & $\mathbf{x}$ & $\mathbf{x}$ & \\
\hline Lasioglossum glabriusculum (MORAWITZ, 1872) & & $\mathbf{x}$ & \\
\hline Lasioglossum laevigatum (KIRBY, 1802) & & $\mathbf{x}$ & \\
\hline Lasioglossum laticeps (SCHENCK, 1868) & $\mathbf{x}$ & $\mathbf{x}$ & \\
\hline Lasioglossum lativentre (SCHENCK, 1853) & $\mathbf{x}$ & $\mathbf{x}$ & \\
\hline Lasioglossum leucopus (KIRBY, 1802) & $\mathbf{x}$ & $\mathbf{x}$ & $\mathbf{x}$ \\
\hline Lasioglossum leucozonium (SCHRANK, 1781) & $\mathbf{x}$ & $\mathbf{x}$ & \\
\hline Lasioglossum cf. limbellum (MORAWITZ, 1876) & $\mathbf{x}$ & & \\
\hline Lasioglossum lineare (SCHENCK, 1868) & & $\mathbf{x}$ & \\
\hline Lasioglossum cf. lucidulum (SCHENCK, 1861) & & $\mathbf{x}$ & \\
\hline Lasioglossum malachurum (KIRBY, 1802) & $\mathbf{x}$ & $\mathbf{x}$ & \\
\hline Lasioglossum minutulum (SCHENCK, 1853) & $\mathbf{x}$ & & \\
\hline Lasioglossum morio (FABRICIUS, 1793) & $\mathbf{x}$ & $\mathbf{x}$ & \\
\hline Lasioglossum nigripes (LEPELETIER, 1841) & $\mathbf{x}$ & $\mathbf{x}$ & \\
\hline
\end{tabular}


Tableau V. Liste des espèces d'Hyménoptères Anthophiles par famille et par département recensées en Franche-Comté : HALICTIDÉS

\begin{tabular}{l|c|c|c|c|} 
Doubs & Jura & $\begin{array}{c}\text { Haute- } \\
\text { Taône }\end{array}$ & $\begin{array}{c}\text { Territoire } \\
\text { de Belfort }\end{array}$ \\
& $(25)$ & $(39)$ & $(70)$ & $(70)$
\end{tabular}

Lasioglossum nitidulum (FABRICIUS, 1804)

Lasioglossum parvulum (SCHENCK, 1853)

Lasioglossum pauperatum (BRULLÉ, 1832)

Lasioglossum pauxillum (SCHENCK, 1853)

Lasioglossum politum (SCHENCK, 1853)

Lasioglossum punctatissimum (SCHENCK, 1853)

Lasioglossum quadrinotatulum (SCHENCK, 1861)

Lasioglossum quadrisignatum (SCHENCK, 1853)

Lasioglossum rufitarse (ZETTERSTEDT, 1838)

Lasioglossum semilucens (ALFKEN, 1914)

Lasioglossum subfulvicorne (BLÜTHGEN, 1934)

Lasioglossum gr. fratellum (fratellum ou subfulvicorne)

Lasioglossum tricinctum (SCHENCK, 1874)

Lasioglossum villosulum (KIRBY, 1802)

Lasioglossum zonulum (SMITH, 1848)

Seladonia confusa (SMITH, 1853)

Seladonia gr. smaragdula

(submediterranea, smaragdula ou orientana)

Seladonia subaurata (RossI, 1792)

Seladonia tumulorum (LinNAEUs, 1758)

Sphecodes albilabris (FABRICIUS, 1793)

Sphecodes crassanus WARNCKE, 1992

Sphecodes ephippius (L., 1767)

Sphecodes ferruginatus HAGENs, 1882

Sphecodes geoffrellus (KIRBY, 1802)

Sphecodes gibbus (L., 1758)

Sphecodes hyalinatus HAGENS, 1882

Sphecodes longulus HAGENs, 1882

Sphecodes monilicornis (KIRBY, 1802)

Sphecodes niger HAGENS, 1874

Sphecodes puncticeps ThOMson, 1870

Sphecodes reticulatus ThOMson, 1870

\begin{tabular}{|c|c|c|c|}
\hline $\mathrm{x}$ & $\mathrm{x}$ & & \\
\hline $\mathrm{x}$ & $\mathrm{x}$ & & \\
\hline & $\mathrm{x}$ & & \\
\hline $\mathrm{x}$ & $\mathrm{x}$ & & \\
\hline$x$ & $\mathrm{x}$ & & \\
\hline $\mathrm{x}$ & $\mathrm{x}$ & $\mathrm{x}$ & \\
\hline$x$ & & & \\
\hline & $\mathrm{x}$ & & \\
\hline $\mathrm{x}$ & $\mathrm{x}$ & & \\
\hline & $\mathrm{x}$ & & \\
\hline $\mathrm{x}$ & & & \\
\hline $\mathrm{x}$ & $\mathrm{x}$ & & \\
\hline & $\mathrm{x}$ & & \\
\hline $\mathrm{x}$ & $\mathrm{x}$ & & \\
\hline$x$ & $\mathrm{x}$ & & \\
\hline & $\mathrm{x}$ & & \\
\hline $\mathrm{x}$ & & & \\
\hline $\mathrm{x}$ & & & \\
\hline $\mathrm{x}$ & $\mathrm{x}$ & & \\
\hline$x$ & $x$ & $\mathrm{x}$ & \\
\hline & $\mathrm{x}$ & & \\
\hline$x$ & $x$ & $\mathrm{x}$ & \\
\hline $\mathrm{x}$ & $\mathrm{x}$ & & \\
\hline$x$ & & & \\
\hline & $\mathrm{x}$ & & \\
\hline & $\mathrm{x}$ & & \\
\hline & & $\mathrm{x}$ & \\
\hline$x$ & $\mathrm{x}$ & $\mathrm{x}$ & \\
\hline $\mathrm{x}$ & $\mathrm{x}$ & & \\
\hline $\mathrm{x}$ & $\mathrm{x}$ & & \\
\hline & $\mathrm{x}$ & & \\
\hline
\end{tabular}


Tableau V. Liste des espèces d'Hyménoptères Anthophiles par famille et par département recensées en Franche-Comté : HALICTIDÉS

\begin{tabular}{l|c|c|c|c} 
Doubs & Jura & $\begin{array}{c}\text { Haute- } \\
\text { Taône }\end{array}$ & $\begin{array}{c}\text { Territoire } \\
\text { de Belfort }\end{array}$ \\
& $(25)$ & $(39)$ & $(70)$ & $(70)$
\end{tabular}

Sphecodes ruficrus (ERICHSON, 1835)

Sphecodes scabricollis WESMAEL, 1835

Région $=63$ espèces

\begin{tabular}{c|c} 
Doubs & Jura \\
(25) & $(39)$
\end{tabular}

(70)

(70)

\begin{tabular}{|c|c|c|} 
& $\mathrm{x}$ & \\
\hline $\mathrm{x}$ & & \\
\hline 42 & 50 & 9
\end{tabular}

0

Légende : En jaune, les cellules indiquent des taxons non identifiés qui ont été recensés sur la base de femelles seulement, mais dont la détermination à l'espèce suppose l'examen de mâles.

Tableau VI. Liste des espèces d'Hyménoptères Anthophiles par famille et par département recensées en Franche-Comté : MEGACHILIDÉS

\begin{tabular}{|c|c|c|c|c|}
\hline Taxons & $\begin{array}{c}\text { Doubs } \\
\text { Jura }\end{array}$ & $\begin{array}{c}\text { Haute- } \\
\text { Saône }\end{array}$ & $\begin{array}{c}\text { Territoire } \\
\text { de Belfort }\end{array}$ \\
& $(25)$ & $\mathbf{( 3 9 )}$ & $\mathbf{( 7 0 )}$ & $\mathbf{( 7 0 )}$
\end{tabular}

\begin{tabular}{|c|c|c|c|c|}
\hline Aglaoapis tridentata (NYLANDER, 1848) & $\mathrm{x}$ & $\mathrm{x}$ & & \\
\hline Anthidiellum strigatum (PANZER, 1805) & $\mathrm{x}$ & $\mathrm{x}$ & & \\
\hline Anthidium manicatum (L., 1758) & $\mathrm{x}$ & $\mathrm{x}$ & $\mathrm{x}$ & \\
\hline Anthidium montanum MoRAwITZ, 1864 & $\mathrm{x}$ & & & \\
\hline Anthidium oblongatum (ILLIGER, 1806) & $\mathrm{x}$ & $\mathrm{x}$ & & \\
\hline Anthidium punctatum LATREILLE, 1809 & $\mathrm{x}$ & $\mathrm{x}$ & & \\
\hline Chelostoma campanularum (KIRBY, 1802) & $\mathrm{x}$ & $\mathrm{x}$ & & $\mathrm{x}$ \\
\hline Chelostoma distinctum (STOECKHERT, 1929) & & $\mathrm{x}$ & $\mathrm{x}$ & \\
\hline Chelostoma florisomne (L., 1758) & $\mathrm{x}$ & $\mathrm{x}$ & $\mathrm{x}$ & \\
\hline Chelostoma rapunculi (LEPELETIER, 1841) & $\mathrm{x}$ & & & \\
\hline Coelioxys afer LEPELETIER, 1841 & $\mathrm{x}$ & $\mathrm{x}$ & $\mathrm{x}$ & \\
\hline Coelioxys alatus FöRSTER, 1853 & & $\mathrm{x}$ & & \\
\hline Coelioxys conoideus (ILLIGER, 1806) & $\mathrm{x}$ & $\mathrm{x}$ & & \\
\hline Coelioxys elongatus LEPELETIER, 1841 & $\mathrm{x}$ & & & \\
\hline Coelioxys lanceolatus NYLANDER, 1852 & $\mathrm{x}$ & & & \\
\hline Coelioxys mandibularis NYLANDER, 1848 & $\mathrm{x}$ & $\mathrm{x}$ & & \\
\hline Coelioxys quadridentatus (L., 1758) & $\mathrm{x}$ & $\mathrm{x}$ & $\mathrm{x}$ & \\
\hline Coelioxys rufescens LEP. \& SERVILLE, 1825 & $\mathrm{x}$ & & & \\
\hline Dioxys cinctus (JURINE, 1807) & $\mathrm{x}$ & & & \\
\hline Heriades truncorum (L., 1758) & $\mathrm{x}$ & $\mathrm{x}$ & $\mathrm{x}$ & \\
\hline Hoplitis adunca (PANZER, 1798) & $\mathrm{x}$ & $\mathrm{x}$ & & \\
\hline
\end{tabular}


Tableau VI. Liste des espèces d'Hyménoptères Anthophiles par famille et par département recensées en Franche-Comté : MEGACHILIDÉS

\begin{tabular}{|c|c|c|c|c|} 
& Doubs & Jura & $\begin{array}{c}\text { Haute- } \\
\text { Taxons }\end{array}$ & $\begin{array}{c}\text { Territoire } \\
\text { de Belfort }\end{array}$ \\
& $(25)$ & $(39)$ & $(70)$ & $(70)$
\end{tabular}

Hoplitis claviventris (THOMSON, 1872)

Hoplitis leucomelana (KIRBY, 1802)

Hoplitis loti (MORAWITZ, 1867)

Hoplitis papaveris (LATREILLE, 1799)

Hoplitis ravouxi (PÉREZ, 1902)

Hoplitis tridentata (DUFOUR \& PERRIS, 1840)

Hoplitis tuberculata (NYLANDER, 1848)

Hoplitis villosa (SCHENCK, 1853)

Megachile alpicola ALFKEN, 1924

Megachile centuncularis (L., 1758)

Megachile circumcincta (KIRBY, 1802)

Megachile ericetorum LEPELETIER, 1841

Megachile genalis MoRAwITZ, 1880

Megachile lapponica THOMson, 1872

Megachile ligniseca (KIRBY, 1802)

Megachile maritima (KIRBY, 1802)

Megachile nigriventris SCHENCK, 1870

Megachile octosignata NYLANDER, 1852

Megachile parietina (GEOFFROY, 1785)

Megachile pilidens ALFKEN, 1924

Megachile pyrenaea PÉREZ, 1890

Megachile pyrenaica LEPELETIER, 1841

Megachile rotundata (FABRICIUS, 1793)

Megachile versicolor SMITH, 1844

Megachile willughbiella (KIRBY, 1802)

Osmia andrenoides SPINOLA, 1808

Osmia aurulenta (PANZER, 1799)

Osmia bicolor (SCHRANK, 1781)

Osmia bicornis (L., 1758)

Osmia brevicornis (FABRICIUS, 1798)

Osmia caerulescens (L., 1758)

\begin{tabular}{|c|c|c|}
\hline $\mathrm{x}$ & $\mathrm{x}$ & \\
\hline \multirow[t]{6}{*}{$\mathrm{x}$} & $x$ & $\mathrm{x}$ \\
\hline & $\mathrm{x}$ & \\
\hline & $x$ & \\
\hline & $x$ & \\
\hline & $x$ & \\
\hline & $\mathrm{x}$ & \\
\hline $\mathrm{x}$ & $\mathrm{x}$ & \\
\hline$x$ & $\mathrm{x}$ & \\
\hline$x$ & $x$ & \\
\hline $\mathrm{x}$ & $\mathrm{x}$ & \\
\hline$x$ & $\mathrm{x}$ & \\
\hline$x$ & $x$ & \\
\hline \multicolumn{3}{|l|}{$\mathrm{x}$} \\
\hline $\mathrm{x}$ & $\mathrm{x}$ & \\
\hline$x$ & & \\
\hline \multirow[t]{2}{*}{$\mathrm{x}$} & $x$ & \\
\hline & $\mathrm{x}$ & \\
\hline $\mathrm{x}$ & $\mathrm{x}$ & \\
\hline \multirow[t]{2}{*}{$\mathrm{x}$} & $\mathrm{x}$ & $x$ \\
\hline & $\mathrm{x}$ & \\
\hline \multicolumn{3}{|l|}{$\mathrm{x}$} \\
\hline $\mathrm{x}$ & $\mathrm{x}$ & $\mathrm{x}$ \\
\hline $\mathrm{x}$ & $x$ & \\
\hline \multirow[t]{2}{*}{$\mathrm{x}$} & $\mathrm{x}$ & \\
\hline & $\mathrm{x}$ & \\
\hline$x$ & $x$ & \\
\hline $\mathrm{x}$ & $x$ & $\mathrm{x}$ \\
\hline$x$ & $x$ & $\mathrm{x}$ \\
\hline $\mathrm{x}$ & $x$ & \\
\hline$x$ & $x$ & $\mathrm{x}$ \\
\hline
\end{tabular}


Tableau VI. Liste des espèces d'Hyménoptères Anthophiles par famille et par département recensées en Franche-Comté : MEGACHILIDÉS

\begin{tabular}{|c|c|c|c|c|} 
& Doubs & Jura & $\begin{array}{c}\text { Haute- } \\
\text { Taxons }\end{array}$ & $\begin{array}{c}\text { Territoire } \\
\text { de Belfort }\end{array}$ \\
& $(25)$ & $(39)$ & $(70)$ & $(70)$
\end{tabular}

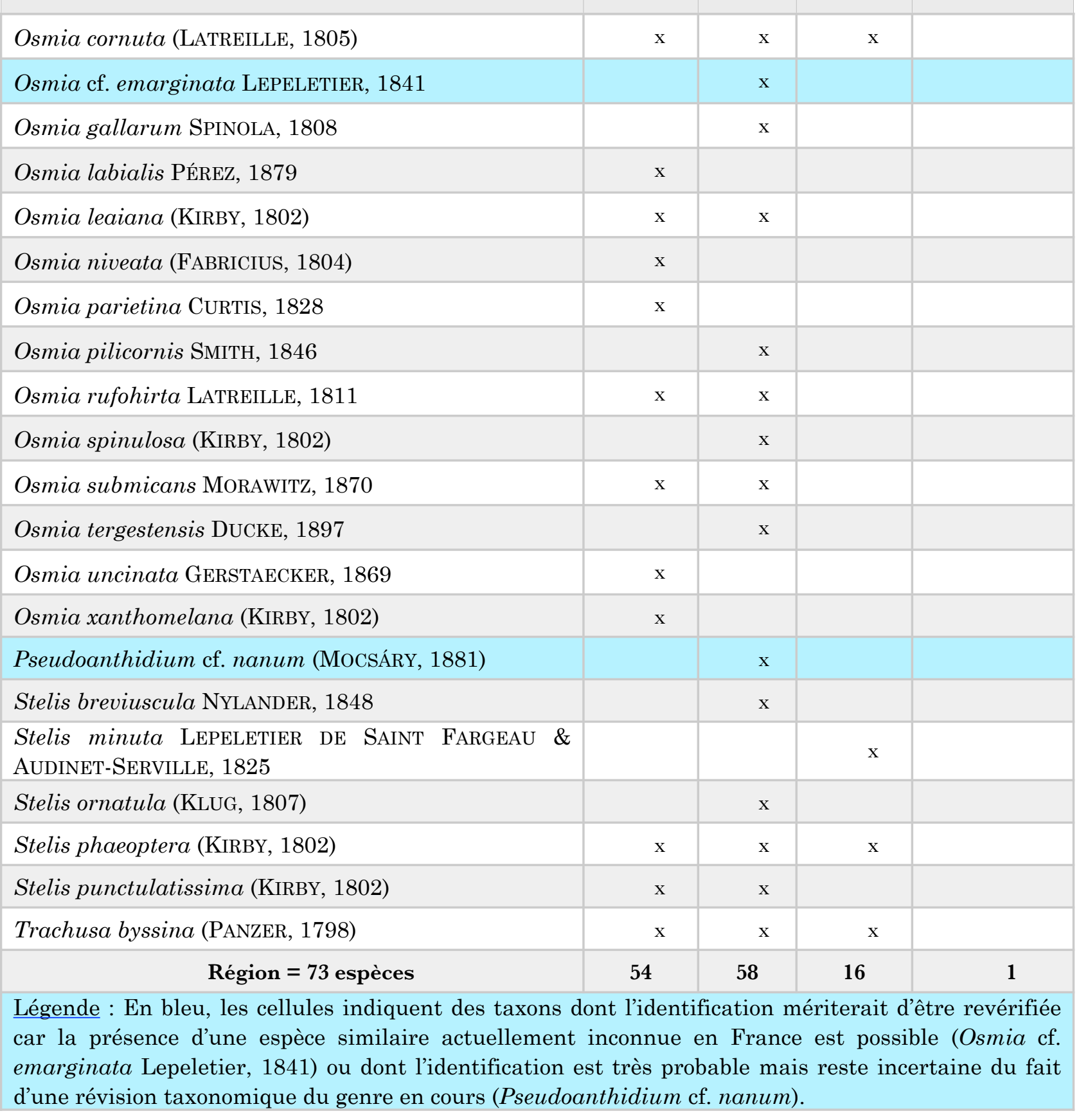


Tableau VII. Liste des espèces d'Hyménoptères Anthophiles par famille et par département recensées en Franche-Comté : MELLITIDÉS

\begin{tabular}{l|c|c|c|c|} 
Taxons & $\begin{array}{c}\text { Doubs } \\
\text { Jura }\end{array}$ & $\begin{array}{c}\text { Haute- } \\
\text { Saône }\end{array}$ & $\begin{array}{c}\text { Territoire } \\
\text { de Belfort }\end{array}$ \\
& $(25)$ & $(39)$ & $(70)$ & $(70)$
\end{tabular}

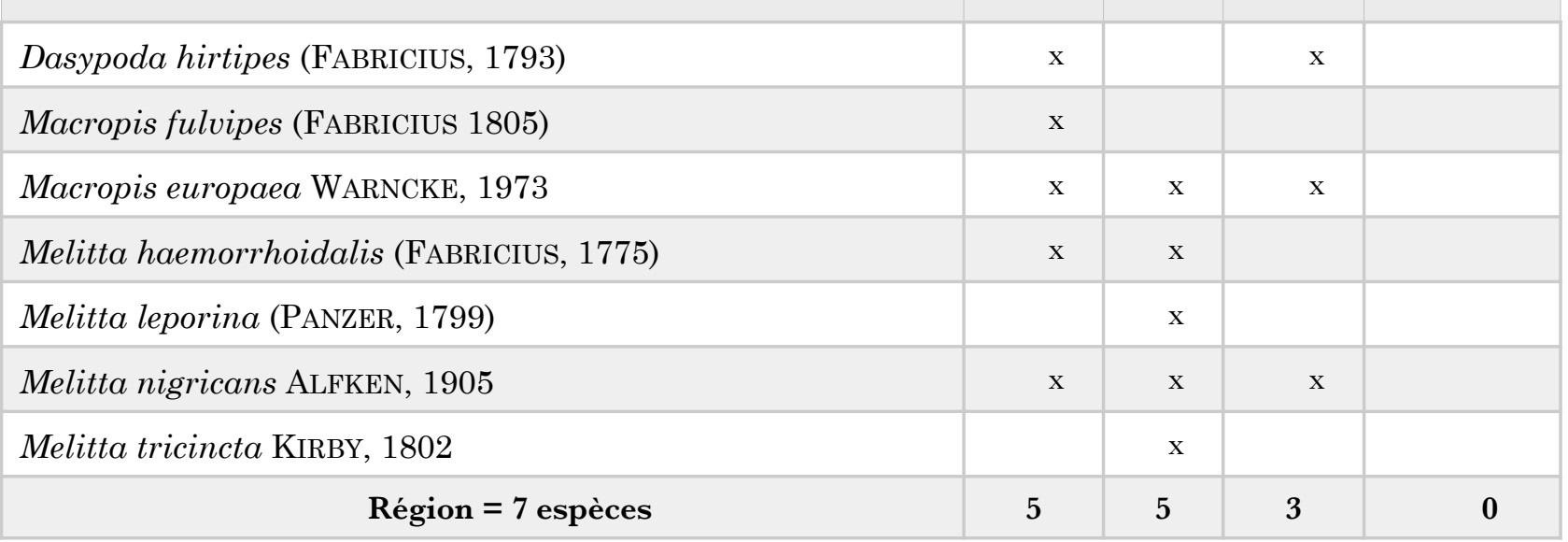

\section{Discussion}

D'autres listes nationales, régionales et départementales existent en Europe. On peut comparer notre synthèse avec celles des 361 espèces évaluées sur la pré-liste rouge d'Alsace (TREIBER, 2015), ou encore avec les 650 espèces de Suisse (RASMONT et al., 2017), pour ne regarder que ce qui existe à proximité. Les 181 espèces inventoriées sur le Grand Dijon (AUBERT, 2018), ou encore les 291 espèces du Grand Lyon (FORTEL, 2014) nous montrent que nous sommes loin d'avoir atteint un palier. Mais nous progressons, au regard du premier comptage réalisé en 2016 sur l'arc jurassien (238 espèces dont 138 coté Ain, 157 coté Jura et 108 coté Doubs) (GENOUd \& MALÉCOT, 2016). La France compte actuellement 972 espèces d'Apoïdes Anthophiles (GENOUD, comm. pers. 2018).

Les limites de ce travail sont liées à la difficulté d'identification des spécimens et à la quantité peu importante de données anciennes et parfois récentes informatisées. Du fait de l'inexistence de bases de données étoffées, nous n'avons pas pu peser la contribution des participants (nombre de données par contributeur). Les localités prospectées sont peu nombreuses en regard de la surface et des habitats de la zone concernée.

Il peut être intéressant d'axer les prospections futures sur la Haute-Saône et tout spécialement le Territoire de Belfort, et dans une moindre mesure sur la montagne jurassienne. Ces secteurs encore mal connus montrent un intérêt important pour d'autres groupes d'Hyménoptères Aculéates non étudiés ici : Apoïdes sphéciformes notamment. Ces départements ont été sous prospectés, ce qui explique le faible nombre d'espèces inventoriées.

De la même manière, réaliser des prospections supplémentaires dans la vallée du Doubs, notamment dans les prairies et sur les berges, la montagne jurassienne, les Vosges, les vallées de la Saône et de l'Ognon peut s'avérer fructueux.

Mais avant toutes choses, la découverte peut se faire à côté de chez nous. C'est sur tous les milieux interstitiels, sur les micro-habitats de talus et de zones dénudées, sur les secteurs particulièrement floricoles qu'il faut prospecter. Dans ces milieux même périurbains, il y a encore beaucoup à découvrir.

\section{Andrenidae}

Avec plus 200 taxons en France et près de 130 en Suisse, il reste encore certainement de nombreuses espèces à détecter, notamment dans le genre Andrena où 43 sont recensées dans l'inventaire.

Apidae

La famille des Apidae (Bourdons, Xylocopes, Cératines, Nomades...) parait bien inventoriée avec le score déjà très intéressant de 69 espèces 


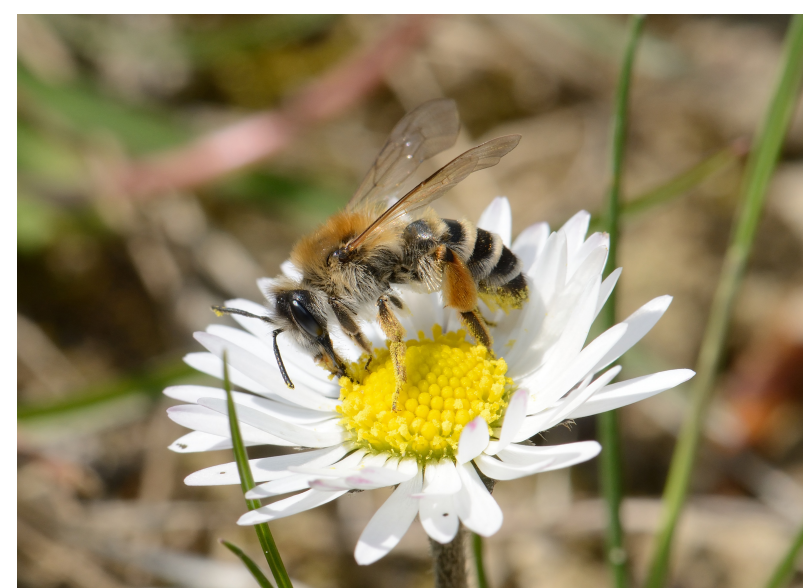

Photo I. Andrena gravida IMHOFF, 1832, femelle. Photo : GenOUd 2019

recensées. Il en reste pourtant encore plusieurs dizaines à découvrir dans la région, notamment dans le genre Nomada, où seules 20 ont été recensées, tandis que 36 taxons sont signalés en en Île-de-France (DUFRÊNE, comm. pers. 2019) et plus de 40 espèces en Hollande (SMIT, 2004).

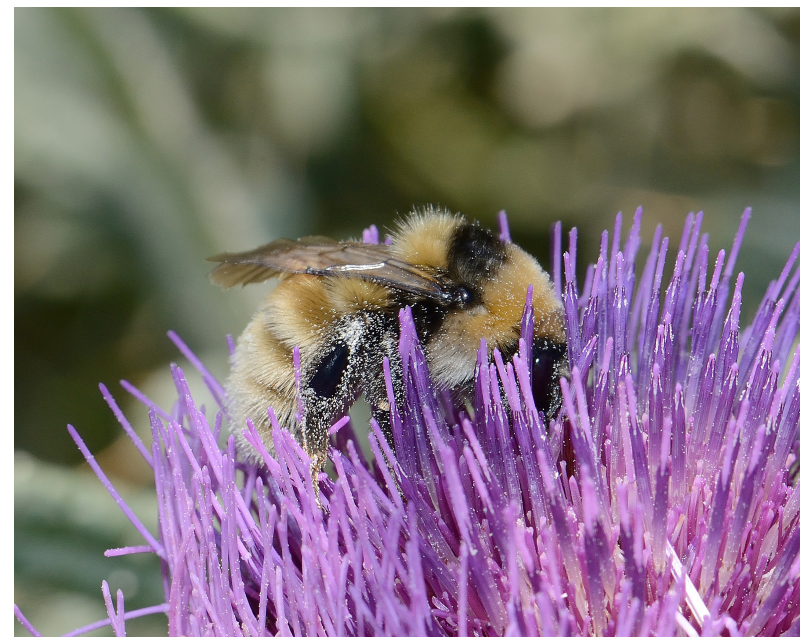

Photo II. Bombus distinguendus MoraWITz, 1869, espèce rare de bourdon observée et identifiée dans le Jura le 5 août 2019 par David GENOUD. Photo : Genoud 2019

La très belle diversité en bourdons, avec 35 espèces recensées, montre bien la bonne conservation des milieux de la montagne jurassienne, laquelle constitue le dernier secteur national pour Bombus distinguendus (photo II), et un refuge pour plusieurs autres espèces devenues très difficiles à rencontrer/détecter, rares et/ou très menacées (modification des paysages et des pratiques agricoles, changement climatique) et dont certaines sont présumées disparues de Suisse (GIRIENS comm pers 2018). On citera Bombus confusus, Bombus pomorum (photo III), Bombus veteranus ou encore Bombus subterraneus parmi les espèces très menacées par l'évolution des pratiques agricoles et Bombus pyrenaeus, Bombus mucidus, Bombus sichelii et Bombus monticola voire Bombus mesomelas, taxons à fortes affinités montagnardes, cantonnés aux crêtes et plus hautes altitudes de la chaîne. Pour ces espèces alticoles, hormis la dernière espèce encore détectable sur la HauteChaîne du Jura nous ne disposons pas de données postérieures aux années 80. Enfin, Bombus muscorum, assez étroitement lié à des milieux humides (landes, marais), est également menacé par le changement climatique drastique (sécheresses périodiques, assèchement des zones humides en période estivale et évolution de la végétation associée). Notons la découverte récente de Tetralonia malvae par Alexandre CORNUEL-WiLleRMOZ (fin juillet et début août 2018) en Haute-Saône et dans le Jura, le long de l'Ognon : cette espèce n'a plus été revue en Suisse depuis l'observation de Jacques DE BEAUMONT en 1954. Des recherches accentuées sur les Anthophores devraient également apporter quelques espèces certainement présentes, telles que Anthophora retusa, A. bimaculata et $A$. quadrimaculata (CHOREIN et al., 2007). A. aestivalis serait à chercher dans le Doubs.

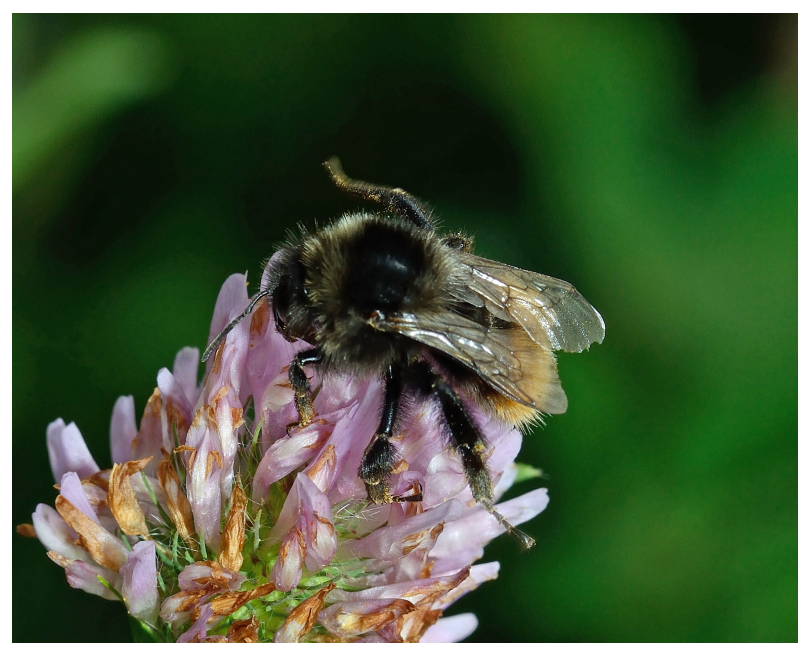

Photo III. Bombus pomorum (PANZER, 1805). Photo : GenOud 2019

\section{Colletidae}

Avec 4 espèces, le genre Colletes est assez bien recensé, il serait néanmoins intéressant de faire des prospections pour certaines plus localisées, C. daviesanus dans le Doubs par exemple, et $C$. fodiens qui n'est pas encore signalée de la région. Le genre Hylaeus ne comprend que 14 espèces, mais d'après GADOUM (comm. pers. 2018), il y en a probablement au moins une trentaine à découvrir. 


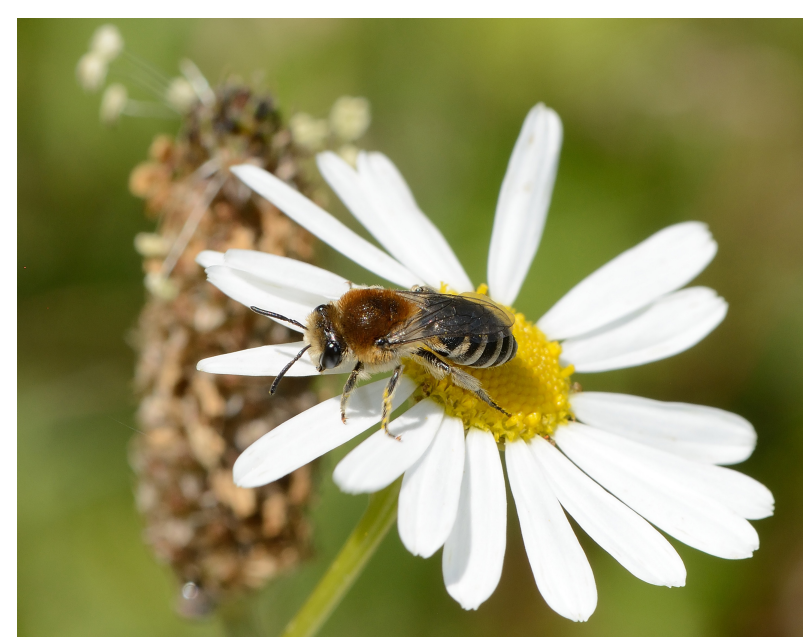

Photo IV. Colletes similis SCHENCK, 1853, femelle. Photo: GenOUd 2019

\section{$\underline{\text { Halictidae }}$}

Le genre Halictus semble assez bien inventorié ; cependant Halictus quadricinctus, la plus grosse espèce du genre, est assez rare, et l'unique mention régionale provient de plusieurs individus capturés dans les années 1970. Les Lasioglossum sont représentés par 34 espèces dans la région. Probablement une vingtaine reste potentiellement à découvrir d'après les ouvrages de PAUlY (2016).

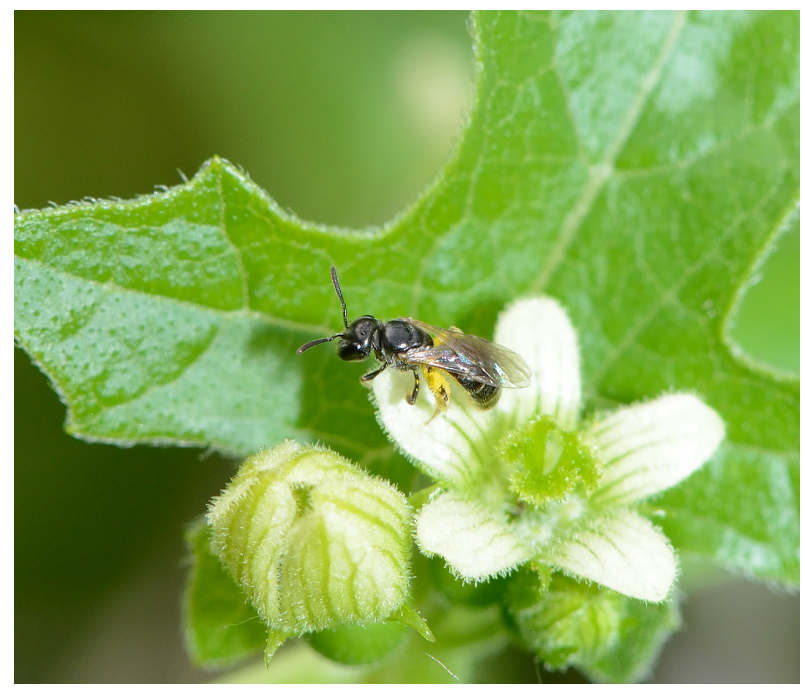

Photo V. Lasioglossum glabriusculum (MORAWITZ, 1872), femelle. Photo : GenOud 2019

Megachilidae

Les Megachilidae constituent une des familles parmi les mieux inventoriées dans la dition avec 73 espèces (sur près de 210 espèces françaises) : ceci s'explique par le fait que Jean-Yves Cretin, et plus récemment Jérome Carminati, se sont particulièrement intéressés à cette famille. De nombreuses espèces rares ont été détectées : citons par exemple Anthidium montanum, taxon boréo-alpin, capturé en août 1976 sur la corniche du Mont d'Or (Doubs), ou Hoplitis tuberculata, dans le Jura en 1977, une espèce montagnarde d'Europe centrale, connue auparavant uniquement dans les Alpes en France. Il resterait à vérifier leur présence actuelle. Parmi les découvertes plus récentes, citons Coelioxys alatus, seconde donnée pour la France (DEvalez 2010), Aglaoapis tridentata capturé à Dole (Jura) en juin 2014 et à Chenecey-Buillon (Doubs) en juin 2018, ou encore Hoplitis loti, une espèce typiquement montagnarde. Le rare et original Megachile genalis, observé déjà dans les années 1970 et 1980 dans les environs de la Station Biologique de Bonnevaux et les tourbières de Remoray, a également été retrouvé ces dernières années par Éric DUFRÊNE.

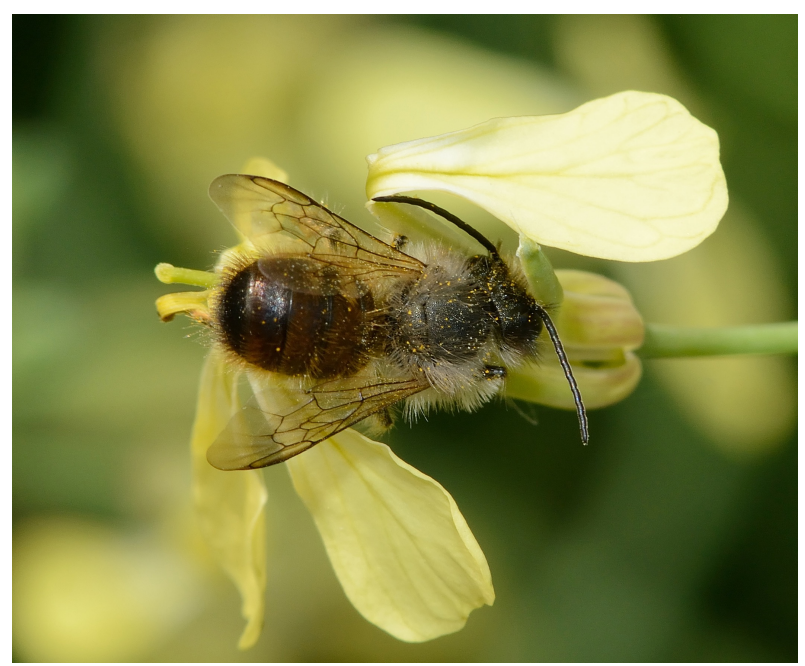

Photo VI. Osmia bicornis (L., 1758), mâle. Photo : Genoud 2019

\section{Melittidae}

La plupart des espèces de la petite famille des Melittidae ont été observées mais ont été recensées avant 1990. Melitta dimidiata serait à rechercher dans la région et la présence actuelle de $M$. tricincta serait à confirmer car elle n'a été contactée que dans les années 1970-1980 par Jean-Yves CRETIN.

\section{Remerciements et participants}

Nous tenons à remercier les membres de l'Observatoire des Abeilles (OA) qui nous ont encouragé dans ce projet, et qui ont participé aux relevés et à l'identification des espèces, mais aussi à la vérification et aux corrections des déterminations. 


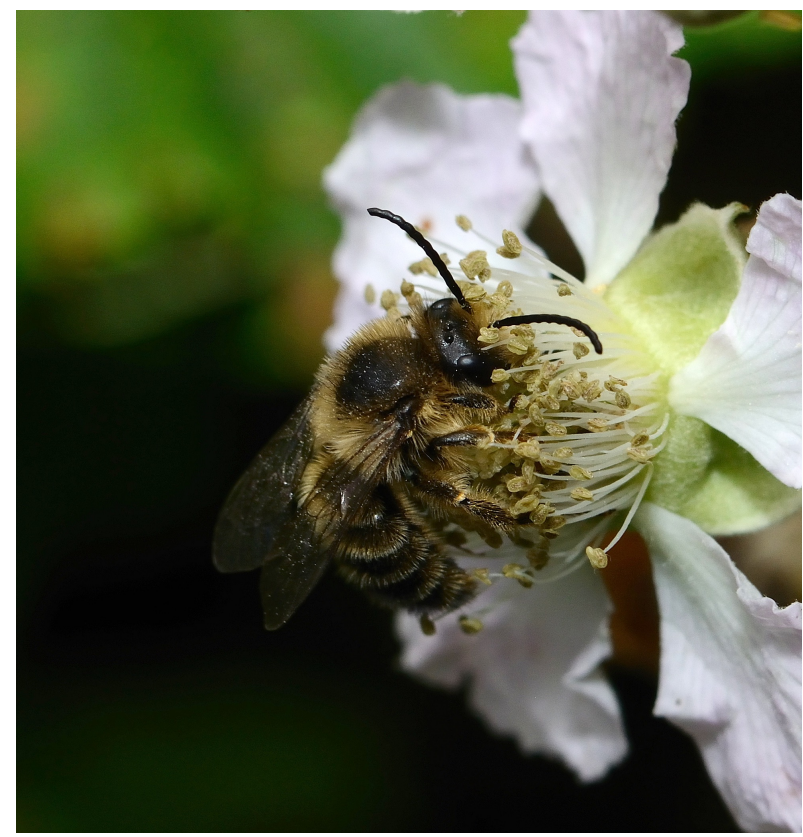

Photo VII. Melitta haemorrhoidalis

(FABRICIUS, 1775), mâle

Photo : GeNOUd 2019

Autres contributeurs validateurs : Holger DATHE, Robert DELMas, Robert FonfRIA $\dagger$, Denis Michez, Alain Pauly, Christophe Praz, Pierre RASMONT, Stephan RISH, Maximilian SchwarZ, Richard VERNIER.

Contributeurs observateurs : Auriane BLIN (LPA Viré), Emmanuel CALHOUN (LEGTA Lons-le-Saunier Mancy), Jérôme CARMInati (OPIE-FC), Henri CHEVIN (INRA Versailles), Jocelyn Claude (RNN du lac de Remoray, Association des Amis de la Réserve Naturelle de Remoray), Mélissande LE CloIREC (LPA Viré), Alexandre Cornuel-Willermoz (OPIE-FC), Bertrand COTTE (OPIE-FC, CEN FC), JeanYves Cretin (OPIE-FC), Catherine Duflot (OPIE-FC), Éric Dufrêne (OA), Camille Favier (LEGTA de Fontaines), Anaïs Fontaine (LEGTA Edgard Faure Montmorot), Serge Gadoum (OPIE), Samia Gareth, (CENFC), David Genoud (OA), Hadrien Gens (RNN du lac de Remoray, Association des Amis de la Réserve Naturelle de Remoray), Dominique LANGLOIS (RNN du ravin de Valbois, CEN-FC), Clémence LAPPRAND (CENFC), Camille MaGand (IUT La Roche sur Yon), Dominique MALÉcot (RNR de la côte de Mancy, LEGTA Lons-le-Saunier Mancy puis CEN-FC), Marin MARMIER (LEGTA Edgard Faure Montmorot), Céline MARTEL (CPIE Bresse du Jura), Frédéric Mora (OPIE-FC), Andreas MÜLLER (ETH Zurich), Jennifer Pichonneau (Lycée d'enseignement agricole privé de Kerplouz-Auray), Christophe PrAz (université de Neuchatel), Jean-Claude ROBERT $\dagger$ (OPIE-FC), Laurence ROSAIN (LEGTA Edgard Faure Montmorot), Jan SMIT (Pays-Bas), Jan SIESs (LEGTA Edgard Faure Montmorot), Philippe STECK $\dagger$ (CLERJ), Pierre Terret (OPIE-FC, et ECOJURA Environnement-Insectes), Camille ThомаS (Université de Saint-Etienne), Bruno Tissot (RNN du lac de Remoray, Association des Amis de la Réserve Naturelle de Remoray).

Nous remercions également Pascal Leblanc pour nous avoir permis et facilité l'accès aux collections du Muséum d'Histoire Naturelle de Besançon.

\section{Bibliographie}

Aubert M, 2018. Abeilles sauvages du Grand Dijon. Coulée verte de l'Ouche depuis le Lac Kir à l'école de gendarmerie, combes et Cras, Année 2017. DREAL Bourgogne Franche-Comté, Dijon Métropole, 43 pp. + annexes.

Berland L, 1925. Faune de France. 10. Hyménoptères Vespiformes 1 (Sphegidae, Pompilidae, Scoliidae, Sapygidae, Mutillidae). Paul Lechevalier, Paris, 364 pp.

Berland L, 1928. Faune de France. 19. Hyménoptères Vespiformes 2 (Eumenidae, Vespidae, Masaridae, Bethylidae, Dryinidae, Embolemidae). Paul Lechevalier, Paris, 208 pp.

Berland L \& Bernard F, 1938. Faune de France. 34. Hyménoptères Vespiformes 3 (Cleptidae, Chrysidae, Trigonalidae). Paul Lechevalier, Paris, 145 pp.

BeRnARD F, 1968. Les Fourmis (Hymenoptera Formicidae) d'Europe occidentale et septentrionale. Masson et Cie, Paris, 411 pp.

Chorein C, Pauly A, \& Rasmont P, 2007. Atlas des Anthophora de Belgique et des régions limitrophes. Atlas Hymenoptera [En ligne]. http://www.atlashymenoptera.net/page.asp?

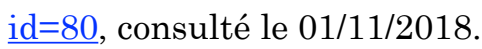

Devalez J, 2010. Nouvelles données sur Coelioxys alata Förster (Hymenoptera Megachilidae), sa biologie et sa distribution. Osmia 4 : 11-14. 
ForTel L, 2014. Écologie et conservation des abeilles sauvages le long d'un gradient d'urbanisation. Thèse de doctorat, Université d'Avignon, INRA d'Avignon, 198 pp.

Genoud D \& LANGLois D, 2013. Inventaire des Hyménoptères Apoïdes de la Réserve Naturelle Nationale du ravin de Valbois. DGE/RNN Ravin de Valbois/FNE Doubs/Observatoire des Abeilles, $32 \mathrm{pp}$.

Genoud D \& Malécot D, 2016. Quelles abeilles sauvages déjà vues sur l'Arc jurassien? L'Azuré 22 : 15.

Hallmann CA, Sorg M, JongeJAns E, SiePel H, Hofland N, SchwAN H \& al., 2017. More than 75 percent decline over 27 years in total flying insect biomass in protected areas. Plos One 12 (10): e $0185809,1-21$. https:// link.springer.com/article/10.1007/ $\underline{\text { s10841-016-9927-1 }}$

Le Féon V, Henry M, Guilbaud L, CoIfFaitGombault C, Dufrêne É, KolodZIEJCZYK E, Kuhlmann M, Requier F \& VAissière B, 2016. An expert-assisted citizen science program involving agricultural high schools provides national patterns on bee species assemblages. Journal of Insect Conservation 20 : 905-918. https://doi.org/10.1007/s10841-016-9927-1

LACHAUSSEE E, 1954. L'équilibre agro-sylvopastoral dans le Jura. Revue Forestière Française 12 : 769-790.

Nieto A, Roberts SPM, Kemp J \& al., 2014. European red list of bees. Publication Office of the European Union [En ligne], Luxembourg. https://ec.europa.eu/environment/nature/ conservation/species/redlist/downloads/ European bees.pdf, consulté le 20/12/2019.

Pauly A, 2016. Le genre Lasioglossum, sousgenre Evylaeus Robertson, 1902, de la Région paléarctique. Atlas Hymenoptera [En ligne]. http://www.atlashymenoptera.net/page.asp? $\underline{\text { ID }=95}$, consulté le 1/11/2018.

Pauly A, 2016. Le genre Lasioglossum, sousgenre Lasioglossum Curtis 1833 en Europe et dans le Bassin Méditerranéen. Atlas Hymenoptera [En ligne]. http:// www.atlashymenoptera.net/page.asp?ID=105, consulté le 1/11/2018.
Rasmont P, Genoud D, Gadoum S, Aubert M, Dufrêne E, Le Goff G, Mahé G, Michez D \& Pauly P, 2017. Hymenoptera Apoidea Gallica : liste des abeilles sauvages de Belgique, France, Luxembourg et Suisse. Atlas Hymenoptera, Université de Mons, Belgique, 15 pp. http://www.atlashymenoptera.net/ bibliogen.asp

RÉAL P, 1987. Les Bourdons du Crêt de la Neige. In Prost J.-F., Réal P., Bordon J., Cornier P., Rolandez J.-L., De Bros F., Robert J.-C, Liogier M., Corcelle J, Roncin P., Siffointe R., Burgunder M., Martin R. \& Contet M., 1987. Contribution à la connaissance biologique de la haute chaîne jurassienne principalement du Crêt de la Neige. Mémoires du Comité de Liaison pour les Recherches Écofaunistiques dans le Jura 3 (2 fascicules) : 216-222.

ROBERT JC, 1967. Étude des hyménoptères aculéates d'une pelouse simili-xérothermique. Éléments de biocénotique. Annales scientifiques de l'université de Besançon (tome. 3B) Écologie $3: 61-68$.

Robert JC, 1992. Le Piège Entomologique Composite (PEC) : une technique d'approche globale des peuplements entomologiques épigés. Bulletin de la Société Entomologique de Suisse 65 : 395-411.

ROBERT JC, 1996. Évaluation d'une technique d'échantillonnage à large spectre de l'entomofaune circulante terrestre : le piège entomologique composite (PEC). Annales de l'Université de Franche-Comté, Besançon, Biologie et écologie 5(4): 13-28.

Sánchez-Bayo F \& Wyckhuys A-G, 2019. Worldwide decline of entomofauna: a review of its drivers. Biological Conservation 232 : 8-27. https://www.sciencedirect.com/science/article/abs/ pii/S0006320718313636

SMIT J, 2004. De Wespbijen (Nomada) van Nederland (Hymenoptera: Apidae). Nederlandse faunistische mededelingen (20): 33-126.

Terret $P$ (COORdonnateur), Tissot $B$, Dierkens M, Mazuy M, Claude J, Speight M, Genoud D, Dufrêne É, Aubert M, Vaissiere B, Artero A \& Mora F, 2012. Compte rendu de l'inventaire entomologique régional bénévole de Franche-Comté de 2011. 
ECOJURA Insectes-Environnement, Non Publié

- ResearchGate, Les Moussières, 30 pp. https:// www.researchgate.net/publication/ 336741799 Compte rendu de l'inventaire bene $\begin{array}{lllllllllllllllll}\mathrm{v} & \mathrm{o} & \mathrm{l} & \mathrm{e} & \mathrm{d} & \mathrm{e} & \mathrm{F} & \mathrm{r} & \mathrm{a} & \mathrm{n} & \mathrm{c} & \mathrm{h} & \mathrm{e} & -\end{array}$

Comte de 2011 complements 2014

Treiber R, 2015. La Liste rouge des Apidés menacés en Alsace. ODONAT, Document numérique version 2, $23 \mathrm{pp}$.

\section{Communications personnelles}

DUFRÊNE É, comm pers, 2019, Observatoire des Abeilles (OA) - 68 rue du Onze Novembre, 59148 Flines-lez-Raches, France

GADOUM S, comm pers, 2018, Office pour les Insectes et leur Environnement (OPIE) - BP 30, 78041 Guyancourt Cedex, France

GENOUD D, comm pers, 2018, Observatoire des Abeilles (OA) - 68 rue du Onze Novembre, 59148 Flines-lez-Raches, France

GIRIENS S, comm pers, 2018

Praz C, comm pers, 2019, Université de

Neuchâtel - Avenue du Premier-Mars 26, 2000

Neuchâtel, Suisse 